\title{
A pragmatic evaluation of sleep patterns before gynecologic surgery
}

\author{
Boaz Sheizaf • Beniamin Almog • Kareemah Salamah • \\ Fady Shehata $\cdot$ Janet Takefman $\cdot$ Togas Tulandi
}

Received: 11 September 2010 / Accepted: 4 November 2010 /Published online: 14 December 2010

(C) Springer-Verlag 2010

\begin{abstract}
The aim of this study was to evaluate preoperative sleep patterns in-patients scheduled for elective endoscopic surgery and to compare sleep characteristics before different types of endoscopic procedures. A prospective survey cohort study (Canadian Task Force classification II-2) was designed to evaluate quantitative and qualitative (score 1-7) sleep parameters. Patients completed sleep-monitoring forms on the baseline (6 weeks before surgery), two nights (night-2), and the night before surgery (night-1). Of a total 119 women, 73 (61\%) underwent laparoscopies and 46 (39\%) hysteroscopies. Forty-seven $(40 \%)$ operations involved organ removal and $72(60 \%)$ were reconstructive surgery. The mean overall sleep-quality score on night-1 $(3.29 \pm 0.15)$ was higher (worse) than on baseline $(2.09 \pm 0.10, P<0.0001)$ and night- $2(2.45 \pm 0.11$, $P<0.001$, respectively). Patients reported more difficulty falling asleep on night-1 $(2.53 \pm 0.15)$ than on baseline $(1.61 \pm 0.09, P<0.0001)$ and night-2 $(1.84 \pm 0.12, P=0.001$, respectively). The mean number of nocturnal awaking events was also highest on night-1 $(1.52 \pm 0.17)$ than on baseline $(0.69 \pm 0.09, P<0.001)$ and night- $2(0.87 \pm 0.09, P<$ 0.05 respectively). Using logistic regression, we found that age, stress level, and time before surgery (baseline, night-2, night-1), were significant predictors of poor sleep. The type of planned surgery did not influence the quality of sleep. Falling asleep on night-1 took longer than on baseline and night-2. There was no difference in the sleep quality measures between the baseline and night-2. Poor sleep quality is common mainly in the night before surgery. The
\end{abstract}

B. Sheizaf $\cdot$ B. Almog $\cdot$ K. Salamah $\cdot$ F. Shehata $\cdot$ J. Takefman

T. Tulandi $(\square)$

Department of Obstetrics and Gynecology, McGill University,

687 Pine Avenue West,

Montreal, QC, Canada H3A 1A1

e-mail: togas.tulandi@mcgill.ca nature and extent of planned surgery do not affect sleep quality variables.

Keywords Sleep · Gynecologic surgery · Surgery · Preoperative

\section{Background}

Sleep is essential to maintain body energy and for quality of life $[1,2]$. The quality of sleep influences health and day time function [3], and it is independently related to various physiological systems [4]. Sleep deprivation is recognized as a stressor [5], but it can also result from stress.

As surgery is one of the most stressful events physically and mentally, it could be associated with sleep disturbances $[6,7]$. Murphy et al. [5] performed a survey examining perioperative sleep patterns in 110 in-hospital patients who were admitted for abdominal surgery. They found a reduction in the mean duration of sleep before and up to 10 days after surgery. The deficit was mainly due to early awakening.

In another study, Kavey and Ahshuler [8] evaluated sleep patterns of ten elective herniorrhaphy patients. They used continuous polysomnography from night 1 of hospitalization until discharge on day 4 or 5 . The patients showed loss of REM (rapid eye movement) and deep phases of sleep in the first two postoperative nights, with a gradual return toward normal sleep over the hospitalization period.

Gogenur et al. [9] studied 12 patients undergoing laparoscopic cholecystectomy and 15 others undergoing laparotomy. They used a sleep questionnaire starting 4 days before surgery until 4 weeks postoperatively. They concluded that immediately after a laparotomy, total sleep time increased (probably due to the use of opioids) and there was 
a change in diurnal sleep distribution. These alterations were less pronounced after laparoscopy. Sleep pattern was disturbed for 4 weeks after major abdominal surgery but only for 1 week after laparoscopic cholecystectomy. On the other hand, Rosenberg et al. [10] found that among ten patients undergoing laparoscopic cholecystectomy, there was no significant difference in the sleep between the night before operation and the first night after operation.

Those studies focused mainly on postoperative sleep pattern while that before surgery generally served as a baseline. In fact, sleep quality a few days before surgery has never been thoroughly investigated. The objectives of our study were to evaluate and describe preoperative sleep patterns in women undergoing gynecologic endoscopic surgery. Increased knowledge of this subject may direct therapy of perioperative sleep disturbances, hence improving quality of life and patient's satisfaction.

\section{Methods}

We conducted a prospective survey among 119 women scheduled to undergo gynecologic endoscopic surgery for benign condition by a single surgeon over a period of 8 months. Women with a known sleep disorder, psychological or mental illness, or inability to complete the study questionnaire in English or French were excluded from the study. The study was approved by the Institutional Review Board of the Sir Mortimer B. Davis, Jewish General Hospital, Montreal, Quebec.

After recruitment, the participants were asked to complete a baseline Sleep Monitoring Form (SMF) under the direction of one of the investigators (BS or KS). The SMF comprises of three groups of questionnaires:

a) Objective (quantitative) queries consists of estimated time the patient went to bed, time to fall asleep, time of morning waking, and the number of nocturnal waking events. These queries are designed to evaluate the three main types of sleep disturbances: difficulty falling asleep, interrupted sleep, and early morning awaking [11].

b) Subjective queries assesses sleep quality on a visual analog scale and consist of the difficulty of falling asleep, the extent of sleep interruption, overall quality of sleep, and the quality of sleep compared with the typical night-sleep.

c) Subjective queries assessing stress level.

The baseline SMF was completed 6 weeks before surgery in the clinic. The participants were then given the SMF and were asked to complete it at home daily, during morning hours. Initially, we asked the patients to complete the questionnaire starting 6 days before surgery until the morning of surgery. However, our preliminary study in 15 patients showed no difference in the sleep patterns between the baselines and sixth to third preoperative nights. Accordingly, we evaluated only the last two preoperative nights before surgery.

All patients were admitted in the morning of surgery and no patient spent the preoperative nights in the hospital. Surgery was performed by a single primary surgeon (TT).

Statistical analysis Data were analyzed using the StatsDirect software (StatsDirect Ltd, Altrincham, Cheshire, UK) and PASW Statistics v18 (formerly SPSS Statistics, Chicago, IL, USA). We used Shapiro Wilks test to evaluate the distribution of the data. Normally distributed data were analyzed using Student $t$ test, skewed data with MannWhitney $U$ test, and proportion with Chi-square test or Fisher's exact test when appropriate. We calculated the means and standard errors for the quantifiable and qualitative sleep variables along the various stages of the preoperative period (defined as "timing"). The second preoperative night was termed night-2 and the last preoperative night was termed night-1. Sleep quality was evaluated by the questionnaires and by a composite poor sleep end point. Sleep was defined as poor if the score of any one of the quality assessing variables (difficulty of falling asleep, extent of sleep interruption, overall sleep quality) was five or higher, and/or more than two events of nocturnal waking were reported.

In addition, we compared the results by three different subgroups: hysteroscopy vs. laparoscopy, organ removal surgery (hysterectomy, oophorectomy, salpingectomy, salpingo-oophorectomy) vs. reconstructive surgery (myomectomy, cystectomy, polypectomy, tubal re-anastomosis, etc.) and major surgery (hysterectomy, laparoscopic myomectomy) vs. non-major surgery. Multivariate logistic regression was constructed to evaluate the effect of various possible predictors on the composite end point of poor sleep throughout the preoperative period. The variables used in the regression included patient age, type of operation (laparoscopy vs. hysteroscopy), nature of operation (organ removal vs. reconstructive surgery), extent of surgery (major vs. minor), and time (baseline night, night 2 , and night -1 ). $P$ value of less than 0.05 was required to reject the null hypothesis.

\section{Findings}

The mean age of the patients was $40.3 \pm 0.7$ years. Of a total 119 women, 73 (61\%) underwent laparoscopy and 46 (39\%) others underwent hysteroscopy. Forty-seven (40\%) of operations involved organ removal and $72(60 \%)$ others were reconstructive surgeries. Thirty-five $(29 \%)$ of the 
patients had major procedures (hysterectomy, myomectomy), and $84(71 \%)$ had non-major surgeries. Two candidates for participation in the study were excluded due to language difficulties not allowing completion of the SMF. Two eligible patients denied participation. One patient was excluded from the study after recruitment. This was due to a death in the family (the Haiti earthquake) the day before surgery.

Sleep quality parameters The different sleep quality variables on baseline night, night-2, and night-1 are presented in Table 1. The mean overall sleep-quality scores on baseline, night-2, and night- 1 were $2.09 \pm 0.10,2.45 \pm 0.11$, and $3.29 \pm 0.15$ respectively. The score on night-1 was significantly higher (worse) when compared with either the baseline night $(P<0.0001,95 \%$ CI 0 to 1$)$ or night-2 ( $P<$ $0.001,95 \%$ CI 1 to 1$)$.

Additional parameters suggested poorer sleep quality on night-1 compared with baseline and night-2 (Table 1). Twenty-two women had poor sleep on baseline as defined by the composite poor sleep end point. More women slept poorly on night-2 (33) and night-1 (60). Of those patients who slept poorly on baseline, all but six also had poor sleep on night-2, and all but four slept poorly on night-1. Poor sleep was significantly more frequent on night-1 than on baseline $(P \leq 0.001,95 \%$ CI 0 to 0$)$ or night-2 $(P \leq 0.001$, $95 \%$ CI 0 to 0$)$. There was no difference in the sleep quality measures between the baseline and night- 2 .

Quantitative parameters Quantitative sleep variables were also significantly different when night-1 was compared with night-2 and baseline night (Table 2). Compared with baseline and night-2, the time to fall asleep on night-1 was longer and morning awaking time was earlier.

Predictors of poor sleep Table 3 demonstrates the results of the logistic regression evaluating the impact of possible predictors on sleep quality. Timing, age, and stress level before sleep were all significant predictors of poor sleep. The type of planned surgery did not influence the quality of sleep. The same was applicable when laparoscopy was compared with hysteroscopy, organ removal versus reconstructive operations, and major versus less major surgeries.

\section{Discussion}

The results of our study show that sleep quality is only impaired in the very last night before surgery (night-1). The additional main finding is the lack of association between the nature of the planned surgery and preoperative sleep characteristics. When compared to either the baseline or the second pre-operative night (night-2), patients on night-1 reported higher (worse) mean overall sleep-quality score, more difficulty of falling asleep, more interrupted sleep, and higher mean number of nocturnal waking events. Night-1 was also characterized by longer period of falling asleep, earlier awaking, and shorter nightly sleep. The latter could probably be attributed to more frequent use of alarm clocks and to deliberate early rising for preparations before surgery.

To date, there is a paucity of information of preoperative sleep studies in the surgical literature. The few available publications $[5,8,10,12]$, though generally methodologically fair, cannot be directly compared with our research. None of them directly addresses pre-operative sleep pattern as the main study objective. Fortunately, some data is available on the related subject of preoperative anxiety.

Sleep disturbance likely represents preoperative anxiety. This subject has been previously studied and described primarily in the anesthesiology literature. Significant preoperative anxiety was reported in $45 \%$ of in-patients and $38 \%$ of day-care patients [13]. Others reported preoperative anxiety in up to $80 \%$ of patients $[13,14]$. Caumo et al. [15] described risk factors for preoperative anxiety in 592 adults. They found a twofold incidence of preoperative anxiety in female patients when compared with males. Other risk factors included a variety of pre-

Table 1 Sleep quality parameters in the preoperative period of 119 women

\begin{tabular}{|c|c|c|c|c|c|c|}
\hline & \multicolumn{3}{|l|}{ Mean \pm SE } & \multicolumn{3}{|l|}{$P$ value } \\
\hline & Baseline & Night-2 & Night-1 & Baseline vs. night- 2 & Baseline vs. night-1 & Night -2 vs. night- 1 \\
\hline No. of nocturnal waking & $0.69 \pm 0.09$ & $0.87 \pm 0.09$ & $1.52 \pm 0.17$ & 0.1 & 0.0002 & 0.01 \\
\hline Difficulty falling asleep (1-7) & $1.61 \pm 0.09$ & $1.84 \pm 0.12$ & $2.53 \pm 0.15$ & 0.3 & $<0.0001$ & 0.0005 \\
\hline Interrupted sleep (1-7) & $1.73 \pm 0.10$ & $1.95 \pm 0.11$ & $2.94 \pm 0.17$ & 0.2 & $<0.0001$ & $<0.0001$ \\
\hline Overall sleep quality (1-7) & $2.09 \pm 0.10$ & $2.45 \pm 0.11$ & $3.29 \pm 0.15$ & 0.06 & $<0.0001$ & 0.0001 \\
\hline Stress before sleep (1-7) & $1.53 \pm 0.08$ & $2.17 \pm 0.14$ & $3.26 \pm 0.18$ & 0.002 & $<0.0001$ & $<0.0001$ \\
\hline
\end{tabular}

night -1 one night before surgery, night-2 = two nights before surgery, $\mathrm{SE}=$ standard error 
Table 2 Quantitative sleep parameters in the preoperative period of 119 women

\begin{tabular}{|c|c|c|c|c|c|c|}
\hline & \multicolumn{3}{|l|}{ Mean \pm SE } & \multicolumn{3}{|l|}{$P$ value } \\
\hline & Baseline & Night-2 & Night-1 & Baseline vs. night-2 & Baseline vs. night-1 & Night-2 Vs. night-1 \\
\hline Bed time (hh:mm) & $22: 47 \pm 00: 04$ & $23: 12 \pm 00: 06$ & $22: 34 \pm 00: 16$ & 0.002 & 0.3 & 0.07 \\
\hline Falling asleep (hh:mm) & $23: 07 \pm 00: 04$ & $23: 36 \pm 00: 05$ & $23: 24 \pm 00: 13$ & 0.0009 & 0.01 & 0.51 \\
\hline Time to fall asleep (min) & $20 \pm 2$ & $24 \pm 2$ & $38 \pm 5$ & 0.3 & 0.0004 & 0.01 \\
\hline Waking time (hh:mm) & $06: 40 \pm 00: 05$ & $08: 34 \pm 12: 46$ & $05: 31 \pm 00: 14$ & $<0.0001$ & $<0.0001$ & $<0.0001$ \\
\hline Sleep duration (min) & $453 \pm 6$ & $465 \pm 7$ & $367 \pm 8$ & 0.11 & $<0.0001$ & $<0.0001$ \\
\hline
\end{tabular}

Night-1 one night before surgery, Night-2 two nights before surgery, $S E$ standard error. $h$ h hours; mm minutes

existing psychiatric disorders, high education, and poor general health state. A previous surgery decreased the risk for preoperative anxiety.

Contrary to our findings, one study showed that the risk of having anxiety was highest in-patients scheduled to medium surgeries [15]. Whether this discrepancy is related to hospitalization before surgery or the type of surgery is unclear. All our patients underwent minimally invasive operations. Besides hysterectomized patients, others were discharged the day of surgery. Perhaps, the patient's background, personality, and individual perceptions play more important roles than the type of planned surgery. Furthermore, our patients underwent surgery for benign condition. Accordingly, cancer phobia, an important contributor to preoperative stress, was probably non-existent.

The significantly poor sleep only in the very last preoperative night suggests a late psychological change when the threat of surgery becomes imminent. Whether deterioration in sleep quality begins earlier in the preoperative period and gradually becomes more severe remain to be examined in a larger study or using more sensitive testing method such as polysomnography. However, this test has an inherent limit of the sample size, and it is not applicable to the patient's natural environment. Furthermore, it is expensive, time-consuming, and requires skilled personnel for testing and interpretation of data [16]. Polysomnography is suitable for evaluation of patients with certain pathologies such as obstructive sleep apnea or chronic sleeping disorders. It is not the tool of choice for large-scale studies with healthy population like ours. Our study is a pragmatic way to evaluate sleep pattern.

Pre operative sleep disturbances are probably triggered by the same factors producing preoperative anxiety including fear of surgery, anesthesia and death, anticipation of postoperative pain, temporary separation from family or friends, incapacitation, and loss of independence [17]. Poor sleep as a marker of anxiety can possibly predict reactions such as increased intraoperative anesthetic requirements $[14,18]$, more difficult control of postoperative pain [19, 20] and higher or more frequent requirement of self administered analgesia [18]. Patients, who report poor quality of sleep, may also report lower satisfaction with treatment and overall quality of life.

Sleep may have a direct effect on physiological systems, not as an intermediate of stress. Sufficient sleep may be involved in healing processes. Reports suggesting that sleep deprivation per se adversely affect the postoperative period or surgical outcome is scarce. Wright et al. [21] tested the hypothesis that poor sleep the night before surgery would predict heightened postoperative pain. Patients $(n=24)$ scheduled for routine breast-conserving surgical procedures for the diagnosis or treatment of cancer were recruited and wore an actigraphy device providing objective estimation of sleep duration and disruption. Their findings did support. Our study suggests that patients might need sleeping medication the night before surgery. Effective short-term treatment for sleep disturbances is fairly simple and available. Benzodiazepines and other hypnotic drugs have
Table 3 Logistic regression: impact of various variables on poor sleep

Night-1 one night before surgery; Night-2 two nights before surgery

\begin{tabular}{lll}
\hline & $P$ value & $95 \%$ CI \\
\hline Age & 0.005 & $1.017-1.101$ \\
Type of surgery (laparoscopy vs. hysteroscopy) & 0.488 & $0.323-1.714$ \\
Nature of surgery (organ removal vs. reconstructive surgery) & 0.691 & $0.357-1.978$ \\
Extent of surgery (major vs. minor surgery) & 0.535 & $0.623-2.489$ \\
Timing (baseline, night-2, night-1) & 0.001 & $0.260-0.883$ \\
Stress level before going to sleep & 0.000 & $1.196-1.644$ \\
\hline
\end{tabular}


been shown to relieve preoperative anxiety. Other therapeutic measures like relaxation techniques or acupuncture [22-24] may also be effective.

This study's main limitation is the use of questionnaires. Questionnaire-based studies are susceptible to certain biases $[13,25,26]$ and often address quality of life variables which are of subjective nature. The strength of our study is its prospective nature, and it is the first study evaluating preoperative sleep before gynecological operations. To date, there is a paucity of information of preoperative sleep studies in surgical literature.

\section{Conclusions}

We conclude that poor sleep quality is frequent in the night before surgery. The nature and extent of the scheduled surgery do not affect sleep quality variables. We advocate increased awareness of both medical staff and patients to this issue. We suggest further research to examine the value of preoperative sleep therapy, in an effort to improve our care to patients undergoing surgery.

Declaration of interest The authors report no conflicts of interest. The authors alone are responsible for the content and writing of the paper.

\section{References}

1. Shapiro CM, Flanigan MJ (1993) Abc of sleep disorders. Function of sleep. BMJ 306:383-385, Clinical research ed

2. Iliescu EA, Coo H, McMurray MH, Meers CL, Quinn MM, Singer MA, Hopman WM (2003) Quality of sleep and healthrelated quality of life in haemodialysis patients. Nephrol Dial Transplant 18:126-132

3. Alapin I, Fichten CS, Libman E, Creti L, Bailes S, Wright J (2000) How is good and poor sleep in older adults and college students related to daytime sleepiness, fatigue, and ability to concentrate? J Psychosom Res 49:381-390

4. Ferin M (1999) Clinical review 105: stress and the reproductive cycle. J Clin Endocrinol Metab 84:1768-1774

5. Murphy F, Bentley S, Ellis BW, Dudley H (1977) Sleep deprivation in patients undergoing operation: a factor in the stress of surgery. BMJ 2:1521-1522

6. Nishiyama T, Yamashita K, Yokoyama T, Imoto A, Manabe M (2007) Effects of quazepam as a preoperative night hypnotic: comparison with brotizolam. J Anesth 21:7-12
7. Kawahara R, Nishimura S, Taenaka N (2005) Actigraphic assessment of the effects of hypnotics on the night before surgery in relation with the severity of surgery. Masui 54:1263-1267

8. Kavey NB, Ahshuler KZ (1979) Sleep in herniorrhaphy patients. Am J Surg 138:683-687

9. Gogenur I, Rosenberg-Adamsen S, Kiil C, Kjaersgaard M, Kehlet H, Rosenberg J (2001) Laparoscopic cholecystectomy causes less sleep disturbance than open abdominal surgery. Surg Endosc 15:1452-1455

10. Rosenberg-Adamsen S, Skarbye M, Wildschiodtz G, Kehlet H, Rosenberg J (1996) Sleep after laparoscopic cholecystectomy. Br J Anaesth 77:572-575

11. Panossian LA, Avidan AY (2009) Review of sleep disorders. Med Clin North Am 93:407-425

12. Gogenur I, Bisgaard T, Burgdorf S, van Someren E, Rosenberg J (2009) Disturbances in the circadian pattern of activity and sleep after laparoscopic versus open abdominal surgery. Surg Endosc 23:1026-1031

13. Wetsch WA, Pircher I, Lederer W, Kinzl JF, Traweger C, HeinzErian P, Benzer A (2009) Preoperative stress and anxiety in daycare patients and inpatients undergoing fast-track surgery. Br J Anaesth 103:199-205

14. Maranets I, Kain ZN (1999) Preoperative anxiety and intraoperative anesthetic requirements. Anesth Analg 89:1346-1351

15. Caumo W, Schmidt AP, Schneider CN, Bergmann J, Iwamoto CW, Bandeira D, Ferreira MB (2001) Risk factors for preoperative anxiety in adults. Acta Anaesthesiol Scand 45:298-307

16. Vaughn BV, Giallanza P (2008) Technical review of polysomnography. Chest 134:1310-1319

17. Egan KJ, Ready LB, Nessly M, Greer BE (1992) Selfadministration of midazolam for postoperative anxiety: a double blinded study. Pain 49:3-8

18. Wells JK, Howard GS, Nowlin WF, Vargas MJ (1986) Presurgical anxiety and postsurgical pain and adjustment: effects of a stress inoculation procedure. J Consult Clin Psychol 54:831-835

19. Anderson KO, Masur FT 3 rd (1983) Psychological preparation for invasive medical and dental procedures. J Behav Med 6:1-40

20. Thomas V, Heath M, Rose D, Flory P (1995) Psychological characteristics and the effectiveness of patient-controlled analgesia. Br J Anaesth 74:271-276

21. Wright CE, Bovbjerg DH, Montgomery GH, Weltz C, Goldfarb A, Pace B, Silverstein JH (2009) Disrupted sleep the night before breast surgery is associated with increased postoperative pain. $\mathrm{J}$ Pain Symptom Manage 37:352-362

22. Hulse RS, Stuart-Shor EM, Russo J (2010) Endoscopic procedure with a modified reiki intervention: a pilot study. Gastroenterol Nurs 33:20-26

23. Pritchard MJ (2009) Managing anxiety in the elective surgical patient. Br J Nurs 18:416-419

24. Movafegh A, Alizadeh R, Hajimohamadi F, Esfehani F, Nejatfar M (2008) Preoperative oral Passiflora incarnata reduces anxiety in ambulatory surgery patients: a double-blind, placebo-controlled study. Anesth Analg 106:1728-1732

25. Smyth C (2003) The Pittsburgh sleep quality index. Medsurg Nurs 12:261-262

26. Yi H, Shin K, Shin C (2006) Development of the sleep quality scale. J Sleep Res 15:309-316 